of a pre-existing host-protein was not postulated at that time. The scrapie agent protein was subsequently identified $^{7}$ and, to the surprise of many, later demonstrated to be derived from a normal host cellular protein ${ }^{8}$. It is the scrapie isoform, but not the cellular isoform, which is associated with transmissibility ${ }^{9}$. We $\mathrm{W}^{10}$ and others ${ }^{11}$ have extended Griffith's original model and our view is that the transmissible spongiform encephalopathies are endogenous neurotoxic disorders that can be transmitted by inoculation or ingestion of an abnormally modified form of the normal gene's product, or inherited via a mutation in this gene.

No matter what the transmissible particle is called - agent, virus, virino or prion - it is by all reckoning unique among pathogens. In the prophetic words of Griffith, "...there is no reason to fear that the existence of a protein

\section{Diet and IQ}

SIR - One of my coauthors ${ }^{1}$ has already responded to Blinkhorn's comments ${ }^{2}$ on our study of the relationship between IQ, vitamin intake and scholastic achievement in four groups of schoolchildren in California ${ }^{3}$. A more technical response follows.

In our study, three cohorts of children were given vitamin-mineral supplements based on 50,100 or $200 \%$ of the US recommended daily allowances for three months and were compared with a group given placebos. Blinkhorn criticized the finding that supplements improved nonverbal IQ and scholastic achievement, his main point being that 87 tests might have been made and our results could have been due to chance. But only five make-or-break tests were made, one for each hypothesis we formulated.

As predicted, a significant difference in total non-verbal IQ was found $(P=0.007)$ on the Wechsler intelligence scale for children-revised (WISC-R) using a four-group ANCOVA of 0 against 50 against 100 against 200 as the make-or-break test on non-verbal IQ. Further examination showed that the $100 \%$ cohort caused the four-group ANCOVA to be significant; this cohort did significantly better than the groups taking the placebo $(P<0.001)$, the $50 \%$ formula $(P<0.05)$ and the $200 \%$ formula $(P<0.05)$. But there was no significant difference when comparing 0 against 50 , 0 against 200 , or 50 against 200 . And, as expected in testing the second hypothesis, no significant difference in total verbal IQ was found on the WISC-R when using a four-group ANCOVA.

A third hypothesis was that if there was a significant difference in non-verbal IQ, the cohort(s) who were responsible might do significantly better than the agent would cause the whole theoretical structure of molecular biology to come tumbling down".

Paul E. Bendheim

DAVID C. BOLTON

Department of Molecular Biology,

New York State Institute for Basic Research,

Staten Island,

New York 10314, USA

1. Weissmann, C. Nature 349, 569 (1991)

2. Griffith, J. S. Nature 215, 1043-1044 (1967).

3. Alper. T. et al. Nature 214, 764-766 (1967).

4. Pattison, I. H. \& Jones, K. M. Vet. Rec. 80, 2-9 (1967)

5. Parry, H. B. Heredity 17, 75-105 (1962)

6. Prusiner, S. B. Science 216, 136-144 (1982)

7. Bolton, D. C. et al. Science 218, 1309-1311 (1982)

8. Oesch, B. et al. Cell 40, 735-746, (1985)

9. Bendheim, P. E. et al. J. infect. Dis. 158, 1198-1208 (1988).

10. Bolton, D. C. \& Bendheim, P. E. Ciba Fdn Symp. 135 164-181 (1988)

11. Oesch, B. et at. Ciba Fon Symp. 135, 209-223 (1988)

placebo group on a scholastic achievement test (the comprehensive test of basic skills, CTBS). Accordingly, the cohort given the $100 \%$ formula was compared with the placebo cohort on total CTBS score, which was calculated by summing the 13 subscales and dividing by 13 . The $100 \%$ cohort produced significantly greater gains $(P=0.024)$.

Blinkhorn made two enormous assumptions, resulting in his belief that 87 tests were carried out. First, he assumed that the 23 WISC-R/CTBS subscales were tested, as well as four total scores (full-scale IQ, non-verbal IQ, verbal IQ and total CTBS score). However, only the last three scores were tested full-scale IQ was not examined at all, and the 23 subscales were studied only to see if the differences were confined to selected areas. (The 100\% cohort surpassed the placebo cohort on four out of five of the non-verbal IQ subscales and 13 out of the 13 CTBS scales.)

Blinkhorn's other assumption was that all three of the cohorts given supplements were individually tested against the placebo group for the 23 subscales, four total scores and two groupadministered IQ tests (the matrix analogies test (MAT) and Raven's matrices (RM)). This would indeed have produced 87 tests. But in reality, verbal IQ, non-verbal IQ, MAT and RM were each tested on one four-group ANCOVA, while academic achievement was tested on one two-group ANCOVA to give a total of five tests only, not 87 .

Blinkhorn also wondered why a change of statistical significance was not expected in non-verbal IQ using the two group-administered tests. The standard deviation on these group tests is about 14 times larger than the expected gain, while the standard deviation of the individually administered WISC-R is about twice the expected gain ${ }^{4}$

These tests were used to determine whether non-verbal IQ tests that are administered in a group are reliable enough to pick up slight changes in IQ. The differences on both group tests exceeded the predicted differences, but neither was significant. These results confirmed the need to use individually administered tests, such as the WISC-R, rather than group tests in this type of research.

To assess our claims properly, Blinkhorn felt that presentation of the raw pre- and post-scores was essential. Those tables have now been published, and they show that the groups were not significantly different initially in nonverbal IQ, verbal IQ, CTBS scores, age, sex or race r.5 $^{4,5 e}$ also complained that evidence of marked bimodality was not published. Those data, submitted to the British Medical Journal, show that the only reason why the $100 \%$ cohort differs significantly from the placebo cohort is because 27 of 105 subjects produced a mean gain of 19.6 points rather than the "expected" 6.6 points which would have given the distributions equal means and standard deviations.

It is a pity that most assessments of our study, including Blinkhorn's, have focused on the pros and cons of taking vitamin supplements and have neglected the reason why this research was done. Our previous work showed that when 803 schools implemented diet policies that allowed only nutrient-dense meals, scholastic achievement rose $16 \%$ for 1.1 million children ${ }^{6}$. The number of children performing two or more grades below expectations fell from 124,000 to 49,000 . A positive correlation between food consumption and achievement occurred after these diet policies were implemented, whereas a negative correlation existed before. We conducted the study because the only way of determining whether better eating habits can have a major effect on performance was to create a placebo-controlled, doubleblind trial involving supplements.

My conclusion from our study is straightforward-eat smart to be smart, but if that is not possible a good supplement set at about $100 \%$ of the US recommended daily allowances makes sense as an insurance policy. For some children, the results will be spectacular.

STEPHEN SCHOENTHALER

California State University, Stanislaus, 801 West Monte Vista Avenue, Turlock, California 95380, USA

\footnotetext{
1. Eysenck, H. J. Nature 351, 263 (1991)

. Blinkhorn, S. Nature 350, 13 (1991)

3. Schoenthaler, S.J., Amos, S.P., Eysenck, H.J., Peritz, E. \& Yudkin, J. Personal. Individ. Diff. 12, 351-362 (1991) 4. Schoenthaler, S.J. Lancet 339, 728-729 (1991).

5. Schoenthaler, S.J. Br. med. J. 302, 1020-1021 (1991) 6. Schoenthaler, S.J., Doraz, W. \& Wakefield, J. Int. J. Biosocial Res. 8, 185-195 (1986)
} 